\title{
Identification of early genetic changes in well-differentiated intramucosal gastric carcinoma by target deep sequencing
}

\author{
Takashi Yoshida ${ }^{1}$ Tatsuya Yamaguchi ${ }^{1}$. Shinya Maekawa ${ }^{1}$. Shinichi Takano ${ }^{1} \cdot$ Toru Kuno $^{1} \cdot$ Keisuke Tanaka $^{1}$. \\ Fumihiko Iwamoto $^{1}$. Yuya Tsukui ${ }^{1}$. Shoji Kobayashi ${ }^{1} \cdot$ Yukiko Asakawa $^{1} \cdot$ Hiroko Shindo $^{1} \cdot$ Mitsuharu Fukasawa $^{1}$. \\ Yasuhiro Nakayama ${ }^{1} \cdot$ Taisuke Inoue $^{1} \cdot$ Tomoyoshi Uetake $^{1} \cdot$ Masahiko Ohtaka $^{1} \cdot$ Tadashi Sato $^{1} \cdot$ Kunio Mochizuki $^{2}$. \\ Nobuyuki Enomoto ${ }^{1}$
}

Received: 17 October 2018 / Accepted: 3 January 2019 / Published online: 11 February 2019

(C) The International Gastric Cancer Association and The Japanese Gastric Cancer Association 2019

\begin{abstract}
Background and aims The recent advancement of next-generation sequencing (NGS) has enabled the identification of cancerrelated somatic aberrations in advanced gastric cancer. However, these remain unclear in early gastric cancers, especially in intramucosal gastric cancers.

Patients and methods Thirty-one well-differentiated (tub1) intramucosal gastric cancers obtained by endoscopic submucosal dissection (ESD) from 29 patients were analyzed. After precise collection of tumors and non-tumors from formalin-fixed paraffin-embedded tissues using laser-captured microdissection (LCM), target sequencing analysis of 50 cancer-related genes was performed using an Ion-Proton sequencer.

Results The most frequent hotspot mutation was found in TP53 (17 lesions, 54.8\%) followed by the Wnt-signaling pathway genes, $A P C$ and $C T N N B 1$ (6 lesions, 19.4\%). The mutations in TP53 and the Wnt-signaling genes were mutually exclusive $(p=0.004)$. There was a tendency that $H$. pylori infection $(p=0.082)$ and macroscopic protrusion $(p=0.095)$ was associated with the presence of these mutations. Only 10 lesions (59\%) among 17 lesions with proven TP53 mutations were positive for p53 immunostaining demonstrating the superiority of the mutational analysis. In addition, focal gene amplification of ERBB2 (16\%) was found frequently in these early stage lesions.

Conclusions Using LCM and NGS, mutations in TP53 and the Wnt-signaling pathway were frequently found and were mutually exclusive in the earliest stage of gastric carcinogenesis.
\end{abstract}

Keywords Somatic mutations $\cdot$ NGS $\cdot$ LCM $\cdot$ Well-differentiated intramucosal gastric carcinoma

Electronic supplementary material The online version of this article (https://doi.org/10.1007/s10120-019-00926-y) contains supplementary material, which is available to authorized users.

Shinya Maekawa

maekawa@yamanashi.ac.jp

1 First Department of Internal Medicine, Faculty of Medicine, University of Yamanashi, 1110, Shimokato, Chuo,

Yamanashi 409-3898, Japan

2 Department of Pathology, Faculty of Medicine, University of Yamanashi, 1110, Shimokato, Chuo, Yamanashi 409-3898, Japan

\section{Introduction}

With the recent advent of next-generation sequencing (NGS) techniques, high-throughput analysis of cancer genome abnormalities has become possible for various types of cancer. Multiple aberrations of cancer-related genes and their signaling pathways have been identified through whole-genome sequencing, whole exome sequencing, and target sequencing in gastric cancer [1-4]. This has led to the description of the landscape of gastric cancer-related somatic aberrations. One of the recent achievements of the Cancer Genomic Atlas (TCGA) project was the molecular classification of gastric cancer into four subclasses including: (1) chromosomal instability type; (2) genomically stable type; (3) microsatellite instability type; and (4) EB-virus type [5]. 
However, identifying the molecular events occurring at the initial stages of gastric cancers is indispensable to better understand the molecular mechanism of gastric carcinogenesis. Previous reports focused their analysis on invasive and advanced cancers with few studies describing cancer-related somatic aberrations occurring in early gastric cancer. Moreover, precise collection of cancer cells without the inclusion of non-cancerous surrounding tissue is pivotal for the accurate evaluation of somatic gene aberrations of cancer. However, the previous studies have not used the recently advanced laser-captured microdissection (LCM) technique for sample collection, and thus, the resultant mutation frequency might not be reliable because of non-cancerous tissue contamination.

Endoscopic submucosal dissection (ESD) is an endoscopic technique developed in Japan for the complete resection of early gastric cancer confined to mucosa [6]. Because of its high curability and low invasiveness, ESD is widely performed in Japan and has been gradually introduced in western countries as one of the standards of care in the treatment of early gastric cancer [7]. Since ESD targets early staged intramucosal gastric cancer and the tumor tissues can be obtained in block with the ESD procedure, ESD-resected specimens are suitable for the analysis of cancer-related genes occurring at the very early stage of gastric carcinogenesis.

In this study, to describe landscape of somatic mutations in cancer-related genes in early staged intramucosal gastric cancers, we undertook NGS-based target sequencing analysis of ESD-resected formalin-fixed paraffinembedded (FFPE) specimens using laser-capture microdissection (LCM) technology and the Ion-Proton-based cancer panel platform.

\section{Patients and methods}

\section{Patients and samples}

A retrospective series of 31 well-differentiated formalinfixed paraffin-embedded (FFPE) intramucosal adenocarcinomas of the stomach from 29 patients were included in this study. All cancers were resected by endoscopic submucosal dissection (ESD) at Yamanashi University Hospital from December 2011 to July 2014. Clinicopathologic characteristics are shown in Table 1. The histological classification was based on the Japanese Classification of Gastric Carcinoma: 3rd English edition [8]. H. pylori infection status was determined by the presence of immunoglobulin $\mathrm{G}$ antibody against $H$. pylori in serum, $\mathrm{C}$ urea breath test, or tissue culture of biopsied samples.
Table 1 Clinicopahotologic characteristics of 31 lesions in 29 patients

\begin{tabular}{lc}
\hline & $n(\%)$ \\
\hline Age (years) ${ }^{\dagger}$ & $74(54-88)$ \\
Sex & \\
Male & $22(75.9 \%)$ \\
Female & $7(24.1 \%)$ \\
H. Pylori & \\
Present & $23(74.2 \%)$ \\
Absent & $8(25.8 \%)$ \\
Location & \\
U & $3(9.7 \%)$ \\
M & $12(38.7 \%)$ \\
L & $14(45.2 \%)$ \\
Remnant stomach & $2(6.5 \%)$ \\
Size (mm) & \\
Macroscopic types & $12(6-29)$ \\
Elevated (IIa/IIa+IIc) & \\
Depressed (IIc/IIc+IIa) & $16(51.6 \%)$ \\
Histological classification & $15(48.4 \%)$ \\
tub1 & \\
tub1>tub2 & $27(87.1 \%)$ \\
Ulcerative findings & $4(12.9 \%)$ \\
Present & \\
Absent & $4(9.7 \%)$ \\
\hline & $27(90.3 \%)$ \\
\hline
\end{tabular}

${ }^{\dagger}$ Median (range)

$U$ upper third, $M$ middle third, $L$ lower third

\section{Laser-captured microdissection (LCM) and DNA extraction}

FFPE blocks were continuously cut into $3-\mu \mathrm{m}$-thick sections for the detection of tumor or non-tumor tissues and $8-\mu \mathrm{m}$-thick sections for laser microdissection, and stained with hematoxylin and eosin (HE). Tumor tissue (T), non-tumor tissue (N1), and stromal lymphocytes (N0) were accurately laser-microdissected using the ArcturusXT ${ }^{\mathrm{TM}}$ Laser-Capture Microdissection System (Life Technologies, Carlsbad, CA, USA) from 8 - $\mu$ m-thick section slides referencing 3 - $\mu$ m-thick sections HEstained slides. DNA from the resected tissues was extracted using QIAamp DNA FFPE kit (QIAGEN, Milan, Italy) following the manufacturer's instructions. The quantity and quality of extracted DNA were assessed using a NanoDrop (Thermofisher, Waltham, MA, USA). An example of sample collected using laser-micro dissection (LCM) is shown in Fig. 1. 
Fig. 1 Example of a sample, where DNA was collected using laser-micro dissection (LCM). As shown, tumor duct epitheliums were accurately dissected without stromal lesions

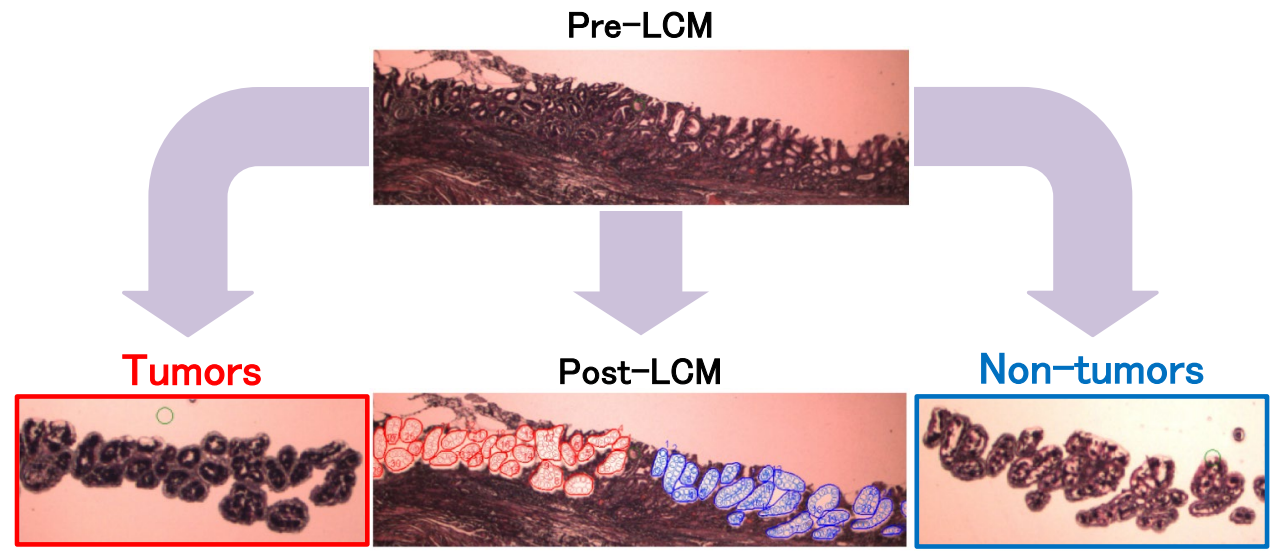

\section{Multiplex PCR of targeted hotspot genes through deep sequencing}

We used ready-made gene panels (Ion AmpliSeq Cancer Hotspot Panel v.2, Life Technologies) to amplify 50 cancer-related target genes. The panel contains 207 primer pairs and targets 2,790 hotspot mutations in the following 50 cancer-related genes in the COSMIC database [9]: $A B L 1, A K T 1, A L K, A P C, A T M, B R A F, C D H 1, C D K N 2 A$, CSF1R, CTNNB1, EGFR, ERBB2, ERBB4, EZH2, FBXW7, FGFR1, FGFR2, FGFR3, FLT3, GNA11, GNAS, GNAQ, HNF1A, HRAS, IDH1, JAK2, JAK3, IDH2, KDR/VEGFR2, KIT, KRAS, MET, MLH1, MPL, NOTCH1, NPM1, NRAS, PDGFRA, PIK3CA, PTEN, PTPN11, RB1, RET, SMAD4, SMARCB1, SMO, SRC, STK11, TP53, and VHL. Briefly, $20 \mathrm{ng}$ of DNA was amplified by polymerase chain reaction (PCR) using these primer panels and the AmpliSeq ${ }^{\mathrm{TM}} \mathrm{HiFi}$ Master Mix (Ion AmpliSeq ${ }^{\mathrm{TM}}$ Library Kit, Life Technologies). The multiplexed amplicons were treated with $\mathrm{FuPa}$ Reagent (Life Technologies) for partial digestion of the primer sequences and phosphorylation. The amplicons were then ligated to adapters from the Ion Xpress ${ }^{\mathrm{TM}}$ Barcode Adapters 1-96 Kit (Life Technologies) according to the manufacturer's instructions. After ligation, the amplicons underwent nick translation and additional library amplification by PCR to complete the linkage between the adapters and amplicons. The size and concentration of the produced amplicon libraries were then checked using the High-Sensitivity DNA Kit (Agilent, Santa Clara, CA, USA) on an Agilent 2100 Bioanalyser on-chip electrophoresis.

Multiplexed barcoded libraries were amplified by emulsion PCR on Ion Sphere ${ }^{\mathrm{TM}}$ particles (ISPs) and sequencing was performed on an Ion Chef ${ }^{\mathrm{TM}}$ System and an Ion-Proton $^{\mathrm{TM}}$ Sequencer (Life Technologies) using the Ion PI Hi-Q Chef Kit (Life Technologies) according to the manufacturer's instructions. The Torrent Suite Software v.4.0 (Life Technologies) was used to align reads to the hg19 reference genome and generate run metrics, including total read counts and quality. Variant Caller v.4.0 (Life Technologies) was used to identify variants.

The cut-off value of variant frequency per read depth was set at $5 \%$. When the variants were detected in $\mathrm{T}$ and not detected in non-tumor tissue, including $\mathrm{N} 0$ and $\mathrm{N} 1$, we defined them as tumor-specific somatic mutations. Variants identified in T, N0, and N1 were defined as non-somatic mutations, which were considered single nucleotide polymorphisms (SNPs) and/or germline mutations.

\section{Focal gene amplification analysis}

To detect possible focal gene amplification, the Ion Reporter plug-in of the dosage quotient (DQ) was used. All .bam files were loaded on the Ion Reporter plug-in and the Confident Germline CNV filter was used to detect CNV variants. To obtain definite results, the final values of 5 or more were defined as focal gene amplification. To confirm the result of focal gene amplification, fluorescent in situ hybridization (FISH) for the ERBB2 and FGFR2 genes was performed in cases judged as focal gene amplification of $E R B B 2$ and FGFR2.

\section{Immunohistochemistry}

For immunostaining, $3 \mu \mathrm{m}$ undyed continuous thin sections of specimens prepared upon DNA extraction were used. MUC5AC (1:50 dilution, NCL-MUC-5AC: Leica, Newcastle, UK), MUC6 (1:50 dilution, NCL-MUC-6: Leica), MUC2 (1:100 dilution, NCL-MUC-2: Leica), CD10 (1:100 dilution, NCL-L-CD10-270: Leica), and p53 (1:50 dilution, M 7001: Dako, Carpinteria, CA, USA) were used as primary antibodies. For antigen activation, autoclave treatment at $120{ }^{\circ} \mathrm{C}$ for 15 min was performed using the Target Retrieval Solution (Dako). Envision TM + Dual Link HRP (Dako) was used as the secondary antibody. After color development with diaminobenzidine, counterstaining with hematoxylin was performed. We classified samples as $\mathrm{p} 53$ positive when 
there was clear positive signal in the nucleus and 30\% of the tumor showed staining. Samples were classified as negative when they displayed weak staining. The classification of mucin phenotype was based on the preliminary report [10]. Samples showing $10 \%$ or more of staining were regarded as positive. Samples that were positive for MUC5AC and MUC6 and negative for both MUC2 and CD10 were classified as gastric type. Samples that were MUC2 and CD10 were positive but MUC5AC and MUC6 negative were classified as intestinal type. Samples positive for MUC5AC, MUC6, MUC2, and CD10 were classified as mixed type. Samples negative for all the markers were defined as unclassified type. These classifications were made blind by two pathologists without previous knowledge of the clinical background or the mutation status. The classification was consistent in these two pathologists.

\section{Statistical analysis}

We compared baseline characteristics of groups by use of the Fisher's exact test for categorical variables. The odds ratios and $95 \%$ confidence intervals were calculated. We used the Cochran-Armitage trend test to detect the trend between gastric mucin phenotypes and somatic mutations in early gastric cancers. $p$ values of $<0.05$ by the two-tailed test were considered to indicate statistical significance.

\section{Results}

\section{Somatic aberrations in cancer-related genes}

The average read depths of N0, N1, and T were 4,661, 5,873, and 5,752, respectively. The median number of mutations identified in N0, N1, and $T$ was 13, 13, and 14, respectively. Supplemental Table 1 shows the read depth and the number of mutations identified in each lesion.

A list of somatic and non-somatic mutations found in 31 lesions is shown in Fig. 2. In Supplemental Table 2, details of somatic mutations identified in 31 lesions are demonstrated. Of the 31 lesions, 24 lesions (77.4\%) had 26 somatic mutations, while somatic mutations were not observed in 7 lesions (22.6\%) (Fig. 2). TP53 was the most common somatic mutation, which was found in 17 lesions (54.8\%). Mutations in the genes of the Wnt signal system (APC, $C T N N B 1)$ were observed in six lesions $(19.4 \%, 3$ lesions in $A P C$ and 3 lesions in $C T N N B 1$ ). In addition, KRAS, NPM1, and $F B X W 7$ mutations were confirmed in one lesion. The NPM1 mutation was observed in the same sample harboring a TP53 mutation, while the $F B X W 7$ mutation was found in the same sample containing the $A P C$ mutation. On the other hand, no lesions harbored both TP53 and Wnt signal gene mutations ( $A P C$ and $C T N N B 1$ ), indicating that those somatic mutations are mutually exclusive in early carcinogenesis ( $p=0.004$, Fisher's direct probability test) (Fig. 2). Besides somatic variants specifically found in $T$, several variants were specifically found in background N0 or N1 (Supplemental Table 1). Though the meaning remains unclear, the role in carcinogenesis might be small, since variant frequency of those variants was low though further studies are needed (variant frequency of $\mathrm{N} 0$ or $\mathrm{N} 1$ was significantly low compared with that of T1).

In $\mathrm{CNV}$ analysis, $E R B B 2$ gain was observed in 5 lesions (16\%), while FGFR2 gain and KIT gain were observed in one lesion (3.2\%), respectively (Fig. 2).

Figure 3 shows a list of 26 mutations. The majority of the identified mutations were point mutations $(n=25,96 \%)$. Of these, missense mutations $(n=14,54 \%)$ were the most frequent, followed by nonsense mutations $(n=9,34 \%)$, splicesite mutation $(n=1,4 \%)$, and stop-loss mutation $(n=1,4 \%)$. As to the pattern of nucleotide substitutions within point mutations, $C: G>T$ :A transition was the most frequent $(14 / 25,56 \%)$ (Fig. 4a). When classifying the $C: G>T: A$ transition according to $5^{\prime}$ and $3^{\prime}$ nucleotide sequences of the mutation, guanine $(G)$ was the most common nucleotide on the $3^{\prime}$ end $(10 / 14,71.4 \%$ ) (Fig. 4b), while $G$ and cytosine $(C)$ were most common on the $5^{\prime}$ end $(6 / 14,42.9 \%)$ (Fig. $4 c$ c).

\section{Risk factor for somatic mutations in TP53/Wnt genes}

To identify the risk factor of having somatic mutations in TP53/Wnt genes, we compared background clinical characteristics between patients having mutations in TP53/Wnt genes $(n=24)$ and patients without $(n=7)$. As shown in Table 2, there was a tendency that $H$. Pylori infection (OR $4.75,95 \%$ CI $0.82-27.50, p=0.082$ ) and macroscopic protrusion (OR 4.67, 95\% CI 0.77-28.40, $p=0.095$ ) were associated with mutations in TP53/Wnt genes.

\section{Mucinous phenotype and mutations in TP53/Wnt genes}

We next investigated the relationship between the mucinous phenotype and cancer-related somatic gene mutations (Supplemental Table 3 ). The mixed type was the most frequently observed phenotype ( $n=23$ lesions, $74.2 \%$ ), followed by the gastric type $(n=5,16.1 \%)$, and the intestinal type $(n=3$, 9.7\%). Gastric cancers with TP53 mutations were found in the gastric type significantly $(p=0.04)$. On the other hand, gastric cancers with the Wnt signal system mutations were not found in the gastric type, but were found only in the intestinal type or mixed type, although the difference did not reach statistical significance $(p=0.21)$. Likewise, no mutation in these cancer-related genes was more frequently found in the intestinal type, although the difference did not reach statistical significance $(p=0.23)$. 


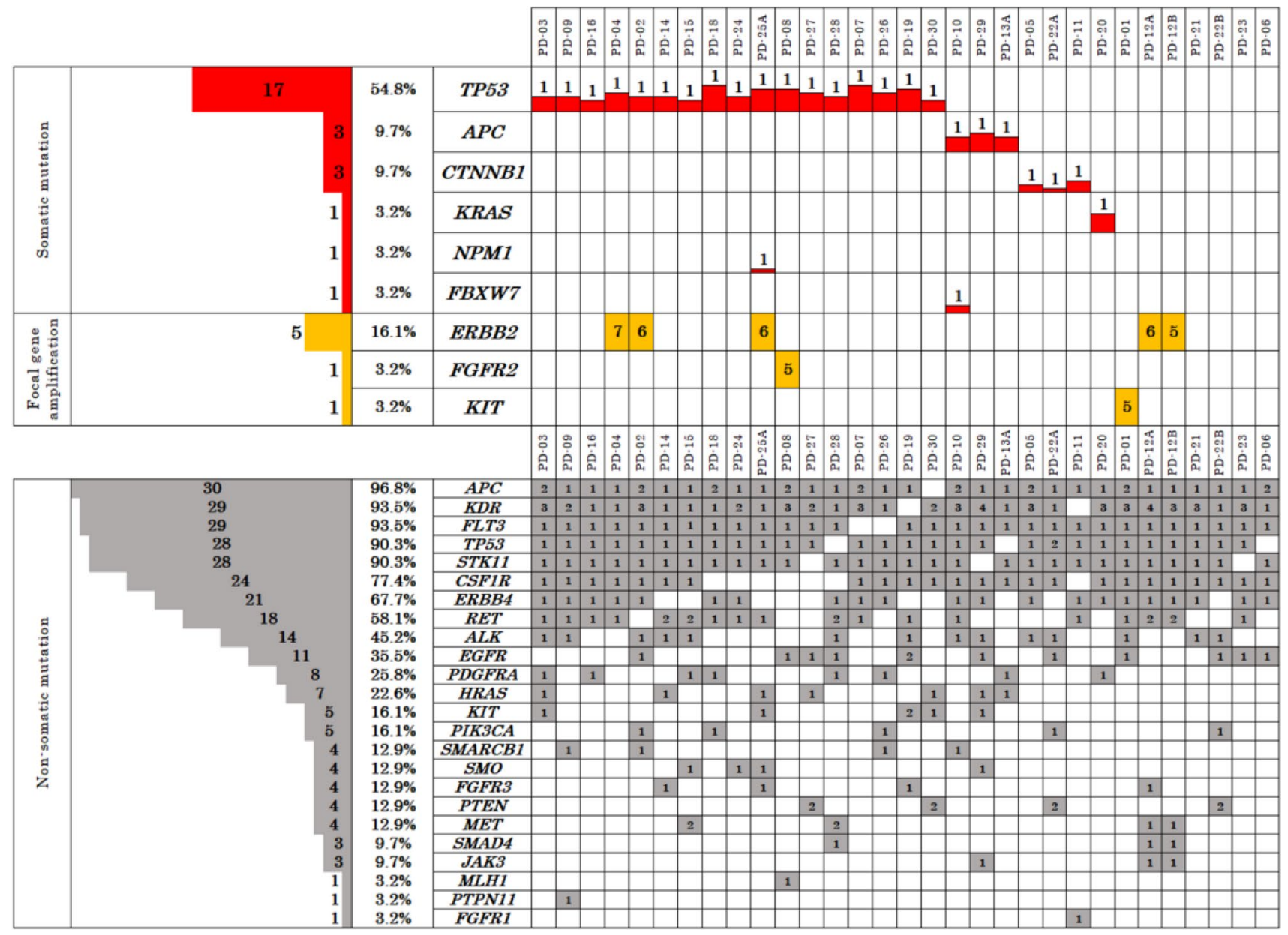

Fig. 2 Profiles of cancer-related genes of the investigated genes in 31 gastric carcinoma lesions. The number of mutations/focal gene amplifications and their rate observed in all 31 lesions are shown on the left axis. In somatic mutations, mutation (variant) frequency in each case was plotted on the right axis (upper panel). The number in each cell (somatic mutations and non-somatic mutations) represents the num-

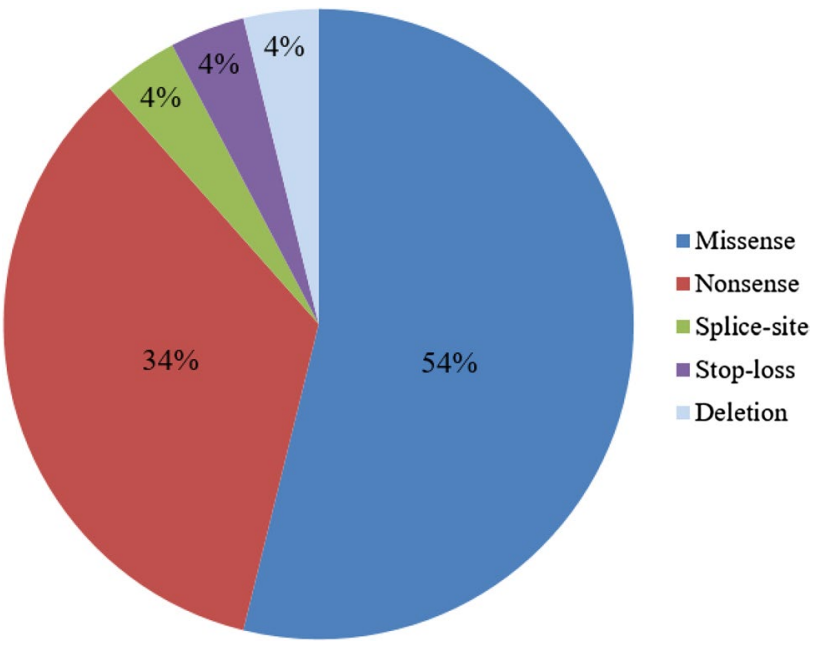

Fig. 3 Twenty-six mutational patterns observed in hotspots in 31 gastric carcinomas ber of mutational loci in each gene. The red bar height represents the variant frequency. The number in each cell (focal gene amplification) represents the values calculated by the Confident Germline CNV filter to detect $\mathrm{CNV}$ variants. The values of 5 or more were considered as focal gene amplification

\section{Comparison between p53 immunostaining and TP53 mutations}

Supplemental Table 4 shows the relationship between p53 immunostaining and TP53 gene mutations. A total of 10 lesions $(32.3 \%)$ were positive for p53 using immunostaining of which all had TP53 gene mutations. Nine out of 10 of the TP53 gene mutations were missense mutations in the DNA-binding domain of TP53, while one lesion was a deletion in the tetrameric oligomerization domain resulting in a frameshift. On the other hand, of the 21 lesions that were negative for p53 immunostaining, TP53 gene mutations were not found in 14 of those lesions. Of the 7 lesions that were positive for the TP53 gene mutations, 6 lesions showed nonsense mutations in the DNA-binding domain, while 1 lesion showed a splice-site mutation. 

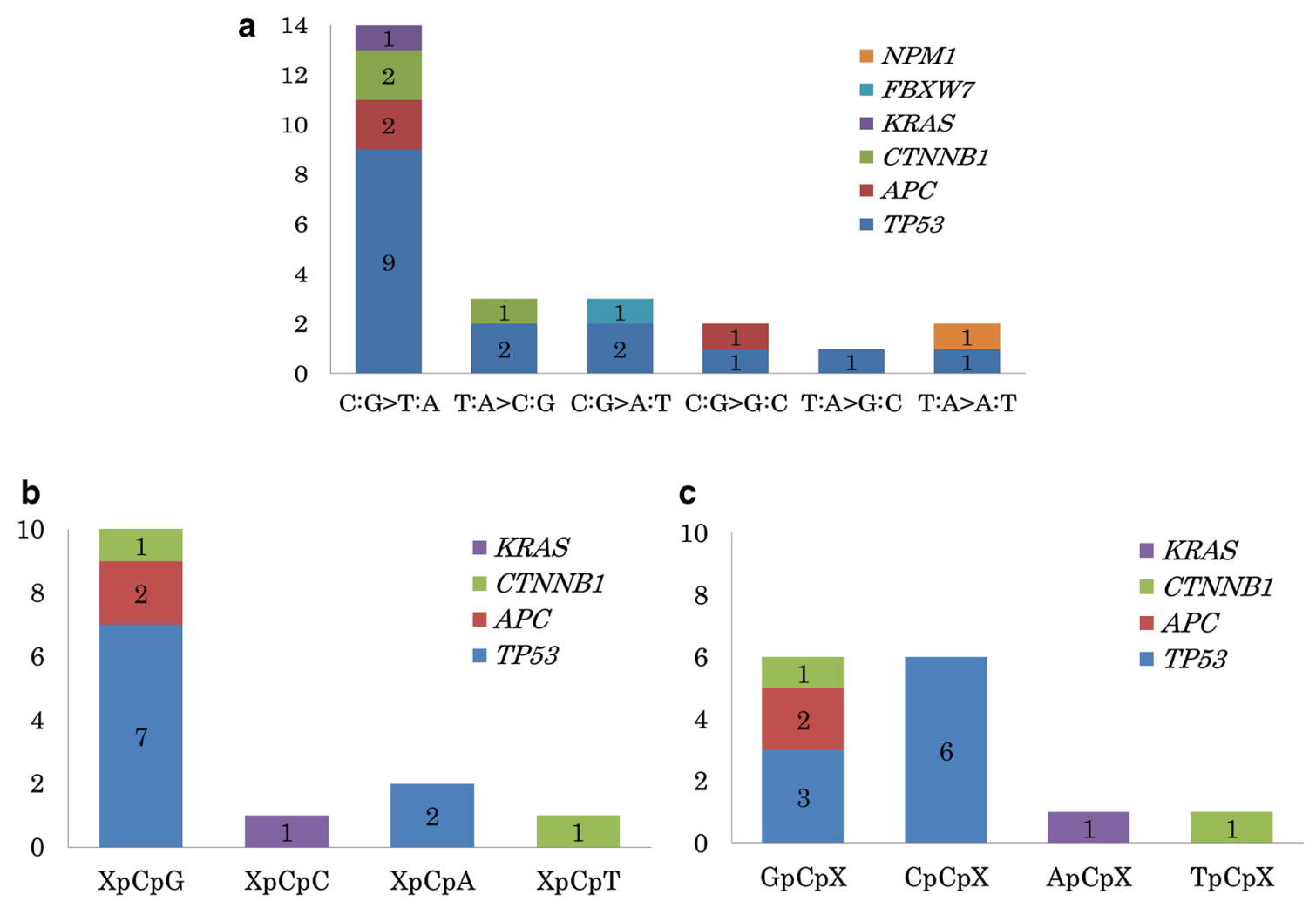

Fig. 4 Mutational signatures of 25 point mutations. The number of mutational signatures classified by the nucleotide substitution pattern is shown (a). Mutational signatures are further classified according to the $5^{\prime}$ (b) or $3^{\prime}$ (c) nucleotides

Table 2 Clinical factors associated with mutations in TP53/Wnt signal genes

\begin{tabular}{|c|c|c|c|c|c|}
\hline & \multicolumn{2}{|l|}{ TP53/Wnt mutation } & \multicolumn{3}{|c|}{ Univariate analysis } \\
\hline & $\begin{array}{l}\text { Present ( } 23 \text { patients, } \\
23 \text { lesions) }\end{array}$ & $\begin{array}{l}\text { Absent ( } 6 \text { patients, } \\
8 \text { lesions) }\end{array}$ & OR & $95 \% \mathrm{CI}$ & $p$ value \\
\hline \multicolumn{6}{|l|}{ Age } \\
\hline 75 years old $\geqq$ & $9(39.1 \%)$ & $3(50.0 \%)$ & 0.64 & $0.11-3.91$ & 0.632 \\
\hline \multicolumn{6}{|l|}{ Sex } \\
\hline Male & $17(73.9 \%)$ & $5(83.3 \%)$ & 0.57 & $0.05-5.88$ & 0.634 \\
\hline \multicolumn{6}{|l|}{ H. pylori } \\
\hline Positive & $19(82.6 \%)$ & $4(66.7 \%)$ & 4.75 & $0.82-27.50$ & 0.082 \\
\hline \multicolumn{6}{|l|}{ Location } \\
\hline $\mathrm{U}$ & $1(4.3 \%)$ & $2(25.0 \%)$ & 0.14 & $0.01-1.77$ & 0.128 \\
\hline \multicolumn{6}{|c|}{ Macroscopic types } \\
\hline Elevated & $14(60.9 \%)$ & $2(25.0 \%)$ & 4.67 & $0.77-28.40$ & 0.095 \\
\hline \multicolumn{6}{|l|}{ Size } \\
\hline $12 \mathrm{~mm}>$ & $8(34.8 \%)$ & $5(62.5 \%)$ & 0.32 & $0.06-1.70$ & 0.181 \\
\hline \multicolumn{6}{|l|}{ Ulceration } \\
\hline Positive & $3(13.0 \%)$ & $1(12.5 \%)$ & 1.05 & $0.09-11.82$ & 0.969 \\
\hline \multicolumn{6}{|c|}{ Mucin phenotypes } \\
\hline Intestinal & $1(4.3 \%)$ & $2(25.0 \%)$ & 0.14 & $0.01-1.77$ & 0.128 \\
\hline
\end{tabular}



a
PD-25A
PD-02
PD-14
PD-12A
PD-12B

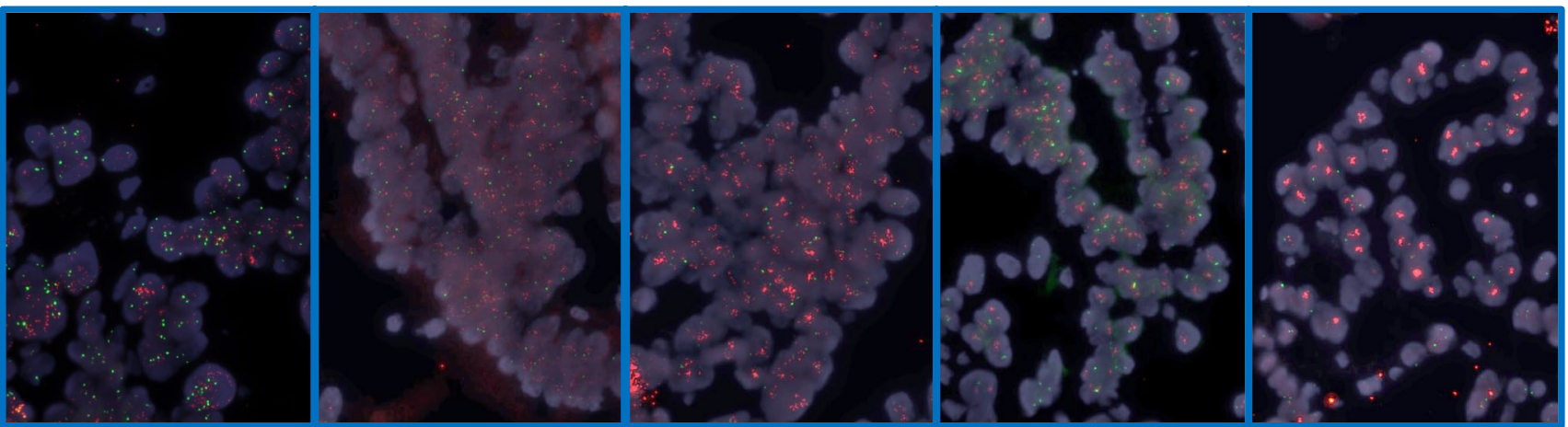

b

PD-08

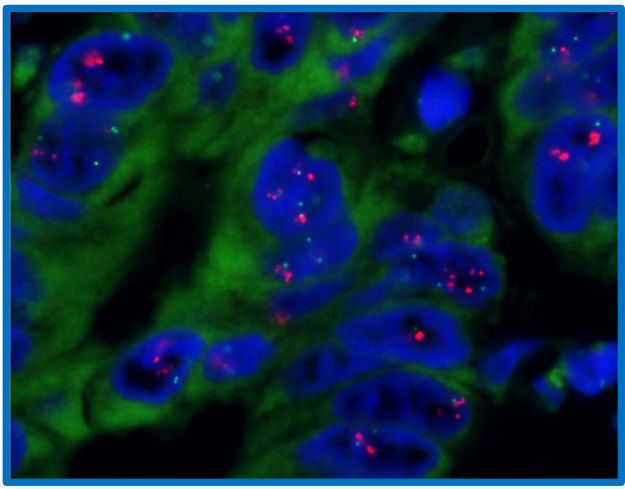

Fig. 5 a FISH analysis of $E R B B 2$ in the five cases judged as $E R B B 2$ gain by mutational analysis. Green signal represents $C E P 17$, while red signal represents ERBB2. b FISH analysis of FGFR2 in one case

\section{Confirmation of ERBB2 and FGFR2 gain by in situ hybridization}

To confirm the ERBB2 and FGFR2 amplification detected by the cancer panel, those cases judged as ERBB2 and FGFR2 gain were subjected to FISH. As shown in Fig. 5, ERBB2 and FGFR2 amplification was confirmed by FISH in all those lesions.

\section{Discussion}

Through the analysis of 31 well-differentiated intramucosal gastric cancer by Ion-Proton-based cancer panel with LCM technology, we showed the frequent presence of mutations in cancer-related genes, including TP53 $(55 \%, 17 / 31)$, Wnt/ $\beta$ catenin (20\%, 6/31 including APC 3/31 plus CTNNB1 3/31), $\operatorname{KRAS}(3 \%, 1 / 31), \operatorname{NPM1}(3 \%, 1 / 31)$, and $F B X W 7(3 \%, 1 / 31)$. We also showed the presence of focal gene amplification in ERBB2 $(16 \%, 5 / 31), F G F R 2(3 \%, 1 / 31)$, and $\operatorname{KIT}(3 \%, 1 / 31)$ in these lesions. In total, cancer-related gene aberrations judged as $F G F R 2$ gain by mutational analysis. Green signal represents $C E P 17$, while red signal represents FGFR2

(hotspot mutations plus focal gene amplification) were present in $87 \%$ of lesions $(27 / 31)$.

The TP53 and Wnt/ $\beta$-catenin genes were the most frequently mutated genes in gastric carcinoma in three previous NGS studies using the Proton platform [11-13]. However, the frequency of the presence of mutations was significantly different in each study. The presence of TP53 mutations was $60 \%$ (9/15), 38\%, (19/50), and 9.7\% (23/238), while the presence of Wnt/ $\beta$-catenin mutations was $20 \%(3 / 15), 4 \%(2 / 50)$, and $0.4 \%(1 / 238)$, respectively, in the studies of Fassan [13], Yoda [11], and Xu [12]. In the studies of Yoda et al. and Xu et al., the studied samples were in most cases advanced and pathologically heterogeneous cancers, including both welldifferentiated and poorly differentiated adenocarcinomas. In addition, the sample collection method was not described. In contrast, in the study of Fassan et al., all samples were well-differentiated early cancers and sample collection was undertaken by "manual" microdissection (though collection accuracy might be somewhat lower compared to "laser-captured" microdissection). Considering the high similarity in the relationship between cancer type and somatic mutation rate between our study and the study of Fassan et al. [13], 
TP53 as well as Wnt/ $\beta$-catenin can be suggested as the main drivers of early staged well-differentiated intramucosal gastric cancer.

Importantly, in spite of the high aberration rate found in this study, the number of hotspot mutations/focal gene amplifications in each cancer was low and confined to only one $(71 \%, 22 / 31)$, two $(13 \%, 4 / 31)$, or three $(3 \%, 1 / 31)$ mutations/focal gene amplifications among thousands of investigated loci. Moreover, the TP53 and Wnt signal mutations $(A P C, C T N N B 1)$ were mutually exclusive in early staged well-differentiated intramucosal carcinoma, indicating that those two genes are both independent and essential drivers in gastric carcinogenesis at the initiation stage, although TP53 and Wnt signal mutations ( $A P C, C T N N B 1)$ might accumulate at the late advanced stage [5].

In contrast, mutations in NPMI and $F B X W 7$ were always accompanied by TP53 and/or Wnt/ $\beta$-catenin mutations. This suggests that these mutations accumulate at later stages of cancer progression. Though the precise role of those two genes in gastric carcinogenesis is unclear, it is speculated that they might enhance carcinogenic signals induced by TP53 and Wnt/ $\beta$-catenin pathways, since NPM1 was reported to associate with the TP53-dependent pathway, while $F B X W 7$ was reported to modulate the $W n t / \beta$-catenin pathway.

Regarding the patterns of nucleotide substitutions, $56 \%$ (14/25) of nucleotide substitutions were $C: G>T$ :A transitions. The result was compatible with a previous study reporting high incidence of $C: G>T$ :A transitions in whole exome sequencing analysis of gastric cancer, more than half of them occurring in the form of $\mathrm{XpCpG}$ trinucleotide sequence $[1,3]$. In recent years, the International Cancer Genome Consortium (ICGC) attempted to classify carcinogenesis factors by analyzing nucleotide substitution patterns called "molecular signature". The $C: G>T:$ A transition in the $\mathrm{XpCpG}$ trinucleotide is considered to occur in association with aging [14]. Given that this was the most frequent mutation found in this study, it could be speculated that aging has a role in gastric carcinogenesis (Fig. 4a). On the other hand, the $C: G>T$ :A transition in the $\mathrm{GpCpX}$ trinucleotide was also frequent (Fig. 4b), suggesting the influence of activation-induced cytidine deaminase (AID), as reported previously.

The analysis of the clinical characteristics suggested an influence of $H$. Pylori infection on TP53/Wnt signal mutations (Table 2). AID is expressed at high frequency in the gastric mucosa associated with $H$. Pylori infection and has been shown to induce mutations in TP53 and CTNNB1 through its enzymatic activity $[15,16]$, resulting in malignant transformation of AID-expressing tissues. In this study, mutated lesions were more common in the macroscopically elevated type. Though there is no previous study reporting the association of elevated type with mutations, it is known that gastric cancer with depressed types frequently develops after $H$. Pylori eradication, and therefore, gastric cancer with elevated type may be a feature associated with active $H$. Pylori infection, though further studies are needed to validate this conclusion.

In this study, an association between the mucin phenotype and cancer-related somatic mutations was suggested: TP53 mutations were recognized in gastric-type lesions, while Wnt-signaling mutations were recognized in intestinal-type lesions (Supplemental Table 3). In the previous studies, the frequency of p53 expression was high in gastric-type cancer [17-19], in agreement with the results shown here disclosing the role of mucin phenotypes in the development of gastric cancer.

Since TP53 was the most frequently mutated gene found here, the status of TP53 mutation was compared with p53 immunostaining. Lesions detected by both methods were missense mutations in the DNA-binding domain or frameshifts in the tetramer organization domain in the TP53 gene. Since tetramer formation is essential for TP53 function and TP53 missense mutations in the DNA-binding domain lacks activity as a transcription factor due to lack of DNA-binding activity, TP53 mutants do not undergo negative feedback by MDM2 resulting in the accumulation of TP53 protein [20]. Of the 21 lesions negative for p53 immunostaining, 7 lesions harbored mutations in TP53. Among these, 6 lesions were nonsense mutations in the DNA-binding domain resulting in non-synthesis of the TP53 protein and thus negative for p53 immunostaining. One lesion showed an intron mutation which might result in a splicing abnormality and thus negativity in immunostaining. p53 immunostaining is used in the diagnosis of gastric cancer. However, more accurate and precise mutation analysis of TP53 should be performed using NGS for clinical applications.

For cases with $A P C$ and $C T N N B 1$ mutations, immunostaining for $\beta$-catenin was added (data not shown). Among three patients with $C T N N B 1$ mutation, two cases showed nuclear accumulation of $\beta$-catenin showing the functional mutational role of $C T N N B 1$ mutation, while $\beta$-catenin accumulation was not observed in three cases with $A P C$ mutation. Since $A P C$ is a tumor suppressor gene, we suspect that the influence on Wnt signal activation might be weaker though further analysis is needed.

In addition, we assessed focal gene amplifications and found the frequent amplification of $E R B B 2, F G F R 2$, and $K I T$. Moreover, ERBB2 amplification was confirmed by FISH. Since copy number aberration of these genes was already reported in advanced gastric intestinal-typed cancers (CIN), it is no surprise to find $E R B B 2$ amplification in the intestinal-typed cancers. On the other hand, several cancers had $E R B B 2$ amplification without any hotpot mutations in TP53 and Wnt/ $\beta$-catenin, indicating the possibility that 
$E R B B 2$ amplification could work as an independent driver. This requires further investigation.

In conclusion, we observed high-frequent cancer-related somatic mutations in well-differentiated early gastric cancer limited to the intramucosa, with mutations in TP53 and Wnt signal genes being the most frequent ones. Analysis of nucleotide substitution patterns resulted in a deeper understanding of the mutational induction in TP53 and Wnt signal genes.

Acknowledgements This study was supported by the Agency for Medical Research and Development (AMED) of Japan.

\section{Compliance with ethical standards}

Conflict of interest We declare that we have no conflict of interest.

Ethical approval This study was approved by the Human Ethics Review Committee of Yamanashi University Hospital.

\section{References}

1. Zang ZJ, Cutcutache I, Poon SL, et al. Exome sequencing of gastric adenocarcinoma identifies recurrent somatic mutations in cell adhesion and chromatin remodeling genes. Nat Genet. 2012;44(5):570-4.

2. Wang K, Yuen ST, Xu J, et al. Whole-genome sequencing and comprehensive molecular profiling identify new driver mutations in gastric cancer. Nat Genet. 2014;46(6):573-82.

3. Wang K, Kan J, Yuen ST, et al. Exome sequencing identifies frequent mutation of ARID1A in molecular subtypes of gastric cancer. Nat Genet. 2011;43(12):1219-23.

4. Nagarajan N, Bertrand D, Hillmer AM, et al. Whole-genome reconstruction and mutational signatures in gastric cancer. Genome Biol. 2012;13(12):R115. (Epub 2012/12/15)

5. Cancer Genome Atlas Research N. Comprehensive molecular characterization of gastric adenocarcinoma. Nature. 2014;513(7517):202-9.

6. Gotoda T. Endoscopic resection of early gastric cancer. Gastric Cancer. 2007;10(1):1-11. (Epub 2007/03/06)

7. Bhatt $\mathrm{A}$, Abe $\mathrm{S}$, Kumaravel $\mathrm{A}$, et al. Indications and techniques for endoscopic submucosal dissection. Am J Gastroenterol. 2015;110(6):784-91. (Epub 2015/01/28)

8. Japanese Gastric Cancer Association. Japanese classification of gastric carcinoma. 3rd english edition. Gastric Cancer. 2011;14(2):101-12. (Epub 2011/05/17)

9. Forbes SA, Bindal N, Bamford S, et al. COSMIC: mining complete cancer genomes in the catalogue of somatic mutations in cancer. Nucleic Acids Res. 2011;39(Database issue):D945-50
10. Shiroshita H, Watanabe H, Ajioka Y, et al. Re-evaluation of mucin phenotypes of gastric minute well-differentiated-type adenocarcinomas using a series of HGM, MUC5AC, MUC6, M-GGMC, MUC2 and CD10 stains. Pathol Int. 2004;54(5):311-21. (Epub 2004/04/17)

11. Yoda Y, Takeshima H, Niwa T, et al. Integrated analysis of cancerrelated pathways affected by genetic and epigenetic alterations in gastric cancer. Gastric Cancer. 2015;18(1):65-76.

12. $\mathrm{Xu} \mathrm{Z}$, Huo $\mathrm{X}$, Ye H, et al. Genetic mutation analysis of human gastric adenocarcinomas using ion torrent sequencing platform. PLoS One. 2014;9(7):e100442.

13. Fassan M, Simbolo M, Bria E, et al. High-throughput mutation profiling identifies novel molecular dysregulation in high-grade intraepithelial neoplasia and early gastric cancers. Gastric Cancer. 2014;17(3):442-9.

14. Matsumoto T, Shimizu T, Takai A, et al. Exploring the mechanisms of gastrointestinal cancer development using deep sequencing analysis. Cancers (Basel). 2015;7(2):1037-51. (Epub 2015/06/18)

15. Shimizu T, Marusawa H, Matsumoto Y, et al. Accumulation of somatic mutations in TP53 in gastric epithelium with Helicobacter pylori infection. Gastroenterology. 2014;147(2):407-17

16. Matsumoto Y, Marusawa H, Kinoshita K, et al. Helicobacter pylori infection triggers aberrant expression of activationinduced cytidine deaminase in gastric epithelium. Nat Med. 2007;13(4):470-6. (Epub 2007/04/03)

17. Tajima Y, Yamazaki K, Makino R, et al. Gastric and intestinal phenotypic marker expression in early differentiated-type tumors of the stomach: clinicopathologic significance and genetic background. Clin Cancer Res. 2006;12(21):6469-79.

18. Sugai T, Tsukahara M, Endoh M, et al. Analysis of cell cyclerelated proteins in gastric intramucosal differentiated-type cancers based on mucin phenotypes: a novel hypothesis of early gastric carcinogenesis based on mucin phenotype. BMC Gastroenterol. 2010;10:55.

19. Sugai T, Inomata M, Uesugi N, et al. Analysis of mucin, p53 protein and $\mathrm{Ki}-67$ expressions in gastric differentiated-type intramucosal neoplastic lesions obtained from endoscopic mucosal resection samples: a proposal for a new classification of intramucosal neoplastic lesions based on nuclear atypia. Pathol Int. 2004;54(6):425-35. (Epub 2004/05/18)

20. Willis A, Jung EJ, Wakefield T, et al. Mutant p53 exerts a dominant negative effect by preventing wild-type $\mathrm{p} 53$ from binding to the promoter of its target genes. Oncogene. 2004;23(13):2330-38.

Publisher's Note Springer Nature remains neutral with regard to jurisdictional claims in published maps and institutional affiliations. 